\title{
A consensus on immunotherapy from the 2017 Chinese Lung Cancer Summit expert panel
}

\author{
Yi-Long Wu ${ }^{1}$, Chang-Li Wang ${ }^{2}$, Mei-Lin Liao ${ }^{3}$, Zhong-Zhen Guan ${ }^{4}$, Chen-Yan Gao ${ }^{5}$, Shun Lu ${ }^{3}$, Ming- \\ Fang Zhao ${ }^{6}$, Jie Wang ${ }^{7}$, Xiao-Qing Liu ${ }^{8}$, Jin-Ji Yang ${ }^{1}$, Jun Liang ${ }^{9}$, Wei-Min Mao ${ }^{10}$, Bao-Hui Han ${ }^{3}$, Xu- \\ Chao Zhang ${ }^{1}$, Yong Song ${ }^{11}$, Ji-Feng Feng ${ }^{12}$, Sheng-Lin $\mathrm{Ma}^{13}$, Gang $\mathrm{Wu}^{14}$, Cai-Cun Zhou ${ }^{15}$, Ke-Neng \\ Chen $^{16}$, Ying Cheng ${ }^{17}$, Yong He ${ }^{18}$, Chun Chen ${ }^{19}$, Qun Wang ${ }^{20}$, Ji-Zhen Lin ${ }^{21}$, Bo Zhu ${ }^{22}$, Yun-Peng Liu ${ }^{6}$, \\ Yi Hu ${ }^{23}$, Gui-Bin Qiao ${ }^{24}$, Qing Zhou ${ }^{1}$, Qi-Bin Song ${ }^{25}$, Nan Wu ${ }^{16}$, Lin Wu ${ }^{26}$, Cheng Huang ${ }^{27}$, Xiao-Long \\ $\mathrm{Fu}^{3}$, Jian-Ping Xiong ${ }^{28}$, Jie $\mathrm{Hu}^{20}$, Cheng-Ping $\mathrm{Hu}^{29}$, Jian-Hua Chang ${ }^{30}$, Qiong Zhao ${ }^{31}$, Jun Zhao ${ }^{16}$, Peng- \\ Hui Zhou ${ }^{4}$, Zhi-Yong Ma ${ }^{32}$, Yuan Chen ${ }^{33}$, He-Long Zhang ${ }^{34}$, Fan Yang ${ }^{35}$, Jian-Jun Wang ${ }^{14}$, Yue-Yin Pan ${ }^{36}$, \\ Xue-Ning Yang ${ }^{1}$, Yun Fan ${ }^{10}$, Zhe Liu ${ }^{37}$, Wen Fan $^{30}$, Nong Yang ${ }^{26}$, Yan-Fang Guan ${ }^{38}$, Hao Sun ${ }^{1}$, Wen-Zhao \\ Zhong'
}

${ }^{1}$ Guangdong Lung Cancer Institute, Guangdong General Hospital and Guangdong Academy of Medical Sciences, Guangzhou 510080, China; ${ }^{2}$ Department of Lung Cancer, Tianjin Medical University Cancer Institute and Hospital, Tianjin 300060, China; ${ }^{3}$ Department of Shanghai Lung Cancer Center, Shanghai Chest Hospital, Shanghai Jiaotong University, Shanghai 200240, China; ${ }^{4}$ Sun Yat-sen University Cancer Center, Guangzhou 510030, China; ${ }^{5}$ Center for Food and Drug Inspection of China Food and Drug Administration, ${ }^{6}$ Department of Medical Oncology, The First Hospital of China Medical University, Shenyang 110001, China; ${ }^{7}$ Department of Medical Oncology, National Cancer Center/Cancer Hospital, Chinese Academy of Medical Sciences and Peking Union Medical College, Beijing 100021, China; ${ }^{8}$ Department of Pulmonary Oncology, 307 Hospital of the Academy of Military Medical Sciences, Beijing 100071, China; ${ }^{9}$ Department of Oncology, Peking University International Hospital, Beijing 102206, China; ${ }^{10}$ Department of Thoracic Surgery, Zhejiang Cancer Hospital, Hangzhou 310000, China; ${ }^{11}$ Department of Respiratory Medicine, Jinling Hospital, Nanjing University School of Medicine, Nanjing 210008, China; ${ }^{12}$ Department of Chemotherapy, Jiangsu Cancer Hospital, Hangzhou 310000, China; ${ }^{13}$ Department of Oncology, Hangzhou First People's Hospital, Nanjing Medical University, Hangzhou 310003 , China; ${ }^{14}$ Department of Oncology, Wuhan Union Hospital, Tongji Medical College, Huazhong University of Science and Technology, Wuhan 430074, China; ${ }^{15}$ Department of Medical Oncology, Shanghai Pulmonary Hospital, Tongji University, Shanghai 200092, China; ${ }^{16}$ Department of Thoracic Surgery I, Peking University Cancer Hospital and Institute, Beijing 100142, China; ${ }^{17}$ Department of Thoracic Oncology, Jilin Provincial Cancer Hospital, Changchun 130012, China; ${ }^{18}$ Department of Respiratory Medicine, Daping Hospital and the Research Institute of Surgery of the Third Military Medical University, Chongqing 400042, China; ${ }^{19}$ Department of Thoracic Surgery, Fujian Medical University Union Hospital, Fujian 350001, China; ${ }^{20}$ Department of Thoracic Surgery, Zhongshan Hospital, Fudan University, Shanghai 200032, China; ${ }^{21}$ Institute of Immunotherapy, Fujian Medical University, Fuzhou 350004, China; ${ }^{22}$ Department of Oncology, Xinqiao Hospital, Third Military Medical University, Chongqing 400030, China; ${ }^{23}$ Department of Internal Oncology, 301 General Hospital, Beijing 100853, China; ${ }^{24}$ Department of Thoracic Surgery, The General Hospital of Guangzhou Military Command, Guangzhou 510010, China; ${ }^{25}$ Cancer Center of Renmin Hospital, Wuhan University, Wuhan 430070, China; ${ }^{26}$ Department of Oncology, Hu Nan Provincial Tumor Hospital, Changsha 410006, China; ${ }^{27}$ Department of Oncology, Fujian Provincial Cancer Hospital, Fuzhou 350011, China; ${ }^{28}$ Department of Oncology, The First Affiliated Hospital of Nanchang University, Nanchang 330039, China; ${ }^{29}$ Department of Respiratory Medicine, Xiangya Hospital, Central South University, Changsha 410008, China; ${ }^{30}$ Department of Medical Oncology, Fudan University Shanghai Cancer Center, Shanghai 200032, China; ${ }^{31}$ Department of Thoracic Oncology, The First Affiliated Hospital, ZheJiang University, Hangzhou 310006, China; ${ }^{32}$ Department of Medical Oncology, Henan Cancer hospital/Affiliated Cancer Hospital of Zhengzhou University, Zhengzhou 450000, China; ${ }^{33}$ Department of Oncology, Tongji Hospital of Tongji Medical College, Huazhong University of Science and Technology, Wuhan 430074, China; ${ }^{34}$ Department of Oncology, Tangdu Hospital, Fourth Military Medical University, Xi'an 710006, China; ${ }^{35}$ Department of Thoracic Surgery, Peking University People Hospital, Beijing 100044, China; ${ }^{36}$ Department of Oncology, Anhui Medical University, Hefei 230032, China; ${ }^{37}$ Beijing Tuberculosis and Thoracic Tumor Research Institute, Beijing Chest Hospital, Capital Medical University, Beijing 100069, China; ${ }^{38}$ Beijing Gene+ Technology, Beijing 102208, China

Contributions: (I) Conception and design: YL Wu; (II) Administrative support: YL Wu; (III) Provision of study materials or patients: All authors; (IV) Collection and assembly of data: YL Wu, H Sun; (V) Data analysis and interpretation: YL Wu, H Sun; (VI) Manuscript writing: All authors; (VII) Final approval of manuscript: All authors.

Correspondence to: Yi-Long Wu. Guangdong Lung Cancer Institute, Guangdong General Hospital and Guangdong Academy of Medical Sciences, Guangzhou 510080, China. Email: syylwu@live.cn. 


\begin{abstract}
The notable clinical success of cancer immunotherapy using checkpoint blockade suggests that it is likely to form the foundation of curative therapy for many malignancies. However, checkpoint blockades do not achieve sustained clinical response in most patients and thus amounts of problems needed to be figured out. Regarding these challenges, the 2017 Chinese Lung Cancer Summit expert panel organized a forum on the 14th Chinese Lung Cancer Summit to formally discuss these controversies. Five consensuses finally were reached to guide the application of checkpoint blockades.
\end{abstract}

Keywords: Lung cancer; immunotherapy; consensus

Submitted Apr 17, 2018. Accepted for publication Apr 19, 2018.

doi: 10.21037/tlcr.2018.04.15

View this article at: http://dx.doi.org/10.21037/tlcr.2018.04.15

\section{Introduction}

The 14th Chinese Lung Cancer Summit was held on 2-3 March 2017, by the Chinese Society of Clinical Oncology and the Chinese Society of Lung Cancer. The theme of the summit was 'Immunotherapy: exploring with hope and perplexity'.

Checkpoint inhibitor has brought a revolution on immunotherapy of cancer as it can bring long-lasting and stable response to multi-tumors, with limited toxicity profile at the same time. Clinical trials also strongly support the antiPD-1/PD-L1 to be the first line therapy for nonsmall cell lung cancer (NSCLC) among patients with high PD-1L express. Even though the checkpoint blocks show impressive results, it's better not to be blindly optimistic until a more reasonable comprehension was set up. There are still such disputes need to be resolved. How to redefine the immunotherapy of lung cancer after this revolution? Are NSCLC patients with oncogene driver mutants suitable to take checkpoint blocks? Which is the most rational strategy to arrange the treatment, monotherapy or in combination therapies? How to choose the most suitable patients group under the background of high economic pressure? As the clinical use of checkpoint blocks is expanding rapidly, how to manage the immunotherapy associated adverse effects in a growing patient population? To solve the divergences above, forming a consensus on immunotherapy in lung cancer is thus in urgent need. During the summit conference, five consensus decisions were achieved regarding current challenges and clinical advisement. The levels of consensus are defined as follows:

(I) Level 1A: consensus based on high-level evidence [rigorous meta-analyses/randomized controlled trials (RCTs)], with the expert panel in unanimous agreement;

(II) Level 1B: consensus based on high-level evidence (rigorous meta-analyses/RCT), with minor controversies among the experts;

(III) Level 2A: consensus based on low-level evidence, with the expert panel in unanimous agreement;

(IV) Level 2B: consensus based on low-level evidence, with minor controversies among the experts;

(V) Level 3: major controversies among the expert panel.

\section{Consensus 1}

\section{The immunotherapy of lung cancer specifically refers immune checkpoint inbibitors (ICIs)}

\section{Level 1B}

Immunotherapies for cancer can be defined as a series of therapies designated to induce immune-mediated destruction against tumor cells. It can be mainly categorized as active, passive or hybrid (active and passive), which include cancer vaccines, oncolytic viruses, adoptive transfer of ex vivo activated T cells, and administration of antibodies or recombinant proteins that regulate the immune system (immune checkpoint blocks etc.) (1).

With the concept that immunotherapy can derive high specificity attack to tumor cells, clinical attempts to use immunological methods in the treatment of human cancer have never been abandoned. Several clinical trials have been designed to verify the efficacy of the immunotherapy like cancer vaccines among lung cancer patients, but few successes were gained. The long-awaited START and MAGRIT study ended with negative results, which suggest that MUC- 1 and MAGE- 3 cannot bring remarkable clinical benefits compared with traditional therapy $(2,3)$.

As many treatment modalities meet their waterloo, recent success of checkpoint blocks brings new hope. Data 
from various clinical trials have proved that monoclonal antibody blocking of cytotoxic $\mathrm{T}$ lymphocyte-associated protein 4 (CTLA-4) and programmed cell death protein 1 (PD1) can achieve sustained clinical response among multitumors. Checkpoint blocks finally break the dawn and boost the development of immunotherapy. The KEYNOTE-024 study directly proved that Pembrolizumab was associated with longer progression-free and overall survival than traditional chemotherapy, which laid the foundation of checkpoint inhibitor as first line therapy for advanced NSCLC with high PD-1L express (4).

The CA184-156 study firstly test efficiency of ipilimumab for lung cancer started from 2012, and then amounts of clinical trials were conducted to further explore its potential effectiveness (5). Nowadays, various of checkpoint blocks are under the clinical experimental stage among Chinese lung cancer patients (PEARL, KN032, NEPTUNE, etc.). The checkpoint blocks will hold the dominant position of immunotherapy for a pretty long time, and further studies will also focus at this area. So, based on these lines of facts, the expert panel reached a consensus that the immunotherapy of lung cancer specifically refers to the checkpoint blocks. And the further study of immunotherapy for lung cancer should also focus on these agents to make more breakthroughs.

\section{Consensus 2}

\section{Checkpoint blocks are not recommended for patients with epidermal growth factor receptor (EGFR) mutation}

\section{Level 1A}

The totally innovation of therapeutic landscape of NSCLC over the last decades relies on the development of EGFR tyrosine kinase inhibitors (TKI). And the concept of precise treatment maximized the clinical benefits of TKIs by limiting the indication to patients with EGFR mutationpositive. Recently, the revolution of immunotherapy for lung cancer enrich the therapeutic paradigms again, it's quite essential to figure out whether the checkpoint blocks should also be recommended to patients with certain oncogene driver mutations.

The realization of "driver mutation" is a breakthrough in targeted therapy. Theoretically, checkpoint blocks target the negative regulatory pathways are associated with immune homeostasis. So, unlike oncogene-targeted TKIs, checkpoint inhibitors rely on promoting an anticancer activity which is not limited to targeting a single oncogenic derangement or other autonomous feature of cancer cells.

The subgroup analysis of several clinical trials for checkpoint blocks also show negative results among patients with oncogene mutations. Keynote- 010 compared the pembrolizumab with traditional docetaxel treatment among patients with previously treated NSCLC, the subgroup analysis shows no significant difference in overall survival between study arms among EGFR-mutant patients (6). The CheckMate-057 also proved that nivolumab and atezolizumab cannot bring more overall survival benefits within the mutations group (7). A retrospective study conducted by Professor Justin et al. directly demonstrate that EGFR mutations and ALK rearrangements are associated with low response rates to PD-1 Pathway Blockade in NSCLC (8). Considering the exist data from various trails, the checkpoint blocks are not recommended as a single therapy for NSCLC patients with oncogene driver mutants.

As the continuous emergence of resistance to the current TKIs, further studies are conducted to find more effective agents. T790M (a threonine-to-methionine substitution at amino acid position 790 in exon 20) is the most common mechanism resistance to EGFR-TKIs. The third generation EGFR-TKIs co-targeted T790M provide an ideal option for NSCLC patients after the resistance (9). But till now, no effective agents are provided for the patients without T790M. The ongoing CheckMate-722 study was designed to verify whether checkpoint block is effective in the treatment of patients with EGFR mutation (T790M negative) NSCLC who failed first line EGFR TKI therapy.

Therefore, the expert panel did not recommend that checkpoint blocks to the be first-line therapy for NSCLC patients with oncogene driver mutants. Further study can be conducted to verify whether checkpoint could be a remediation for patients with EGFR mutation, T790M negative NSCLC.

\section{Update}

Several pre-clinical and epidemiology studies have been conducted till now, but there are still controversies over the application of ICIs in EGFR mutant NSCLC. These controversies are mainly focus on the relationship between PD-1L/PD-1 expression level and EGFR status, clinical benefit of immune checkpoint blocks in EGFR mutant NSCLC, and potential mechanism which impede the immune effect among EGFR mutation patients.

The latest results from several pooled analyses suggest an inverse association between PD-L1 level and EGFRmutation status. But a number of studies come out with 
opposite conclusion which may due to the immature and heterogeneity of detection for PD-1/PD-L1. More convinced data is still required to solve this conflict.

To evaluate the efficacy of AntiPD-1/PD-1L within EGFR mutation NSCLC, two recently published metaanalysis show decreased clinical benefits when compared with traditional chemotherapy. Then several potential underlying mechanisms are proposed to explain the impaired response to antiPD-1/PD-L1. An analysis based on the repository database have proved that EGFR mutation NSCLC is lack in T-cell infiltration and present lower tumor mutation burden (TMB). Another study also showed that EGFR mutation can suppress the immune function by IFN gamma pathway.

Considering the immunodeficiency environment of EGFR mutation NSCLC, several trails try to combine checkpoint blocks with chemotherapy or TKI for improving the efficacy. But these trails also show an increase in the frequency of treatment related adverse interstitial lung disease and hepatitis which caused by the overlapping toxicity of combine therapy. Further study should be conducted to verify a more reasonable treatment schedule.

Bad news from published data have constantly disappointed patients with EGFR mutation NSCLC resisted to TKI who embraced hope for the checkpoint blocks, but we can still see hope from a small group of patients who have achieved partial response and desirable clinical benefits. Further studies are needed for exploring more ideal biomarkers which can multi-dimensionally character the tumor immune environment and identified the potential benefit groups.

\section{Consensus 3}

\section{Checkpoint blocks should be applied to specific groups concerning the economic factors}

\section{Level 1B}

Through incredible advancements in medical technology, especially gene-sequencing technology, precision medicine has already come into reality in some medical settings. Patients can get the treatment tailored to an individual's genetic make-up, which will maximum the therapeutic effects. The most suitable example is the development of TKIs targeted EGFR have brought remarkable clinical benefits for EGFR-mutants NSCLC. Nowadays, checkpoint blocks have burst the development of immunotherapy and can bring long-lasting and stable response to multi-tumors, but it does not achieve sustained clinical response in most patients at the same time. Concerning the economic factors, it is quite necessary to work out effective biomarkers to seek out the groups who would benefit from checkpoint inhibitors.

As the remarkable success in EGFR-TKIs is based on the deep interpretation of EGFR signaling pathway, another breakthrough in immunotherapy should also be supported by a full appreciation of immune environments and associated features. According to PD-L1 status and presence or absence of tumor-infiltrating lymphocytes (TILs), Professor Teng proposed a model which divide the tumor immune environments into four types. These included the type I (adaptive immune resistance, $\mathrm{TIL}^{+} / \mathrm{PD}-\mathrm{L1}^{+}$), type II (immunologic ignorance, $\mathrm{TIL}^{-} / \mathrm{PD}-\mathrm{L1}^{-}$), type III (intrinsic induction, $\mathrm{TIL}^{-} / \mathrm{PD}-\mathrm{L}^{+}$), and type $\mathrm{IV}$ (tolerance, $\mathrm{TIL}^{+} / \mathrm{PD}-$ $\left.\mathrm{L1}^{-}\right)$(10). This simple initial stratification sets a framework to identify the most suitable immunotherapeutic strategies. As the $\mathrm{T}$ cells are sufficient inside the tumor and these $\mathrm{T}$ cells are inducing an adaptive expression of PD-L1, data from human melanoma suggest that type I tumors were most likely be benefit from single-agent anti-PD-1/L1. On the contrary, type II, III and IV tumors are considered to have poor prognosis as their lack of immune reaction or within other suppressive pathways. Further therapeutic strategies included combination therapy should be designed to turn on the immune effects and reverse the immune suppression. This proposal makes it simple and feasible to identify the potential groups who would benefits from the checkpoint blockade, but it also have limitations such as the presence of TIL is not dichotomous variable, and the deviation of PD-1L level tested by tumour biopsies is not avoidable.

To define the phenotypes associated with patients' response to anti-PD-L1/PD-1 therapy, professor Chen also proposed a model which distinguish three basic immune profiles. The first profile was named as "immune-inflamed phenotype", which was characterized by the presence of active $\mathrm{T}$ cells inside the tumour bed. Studies have shown that clinical response to antiPD-1/PD-L1 therapy was most often occurred in the immune inflamed tumors. The second profile was the immune-excluded phenotype, immune cells in which are retained in the stroma that surrounds nests of tumour cells. Clinical responses are uncommon with the second phenotype tumors as the antitumor response was rendered ineffective by a block in tumour penetration through the stroma. The last profile named "immunedesert phenotype", is characterized by a deficient of T cells in either the parenchyma or the stroma of the tumor. 
As the lack of pre-existing antitumour immunity, such tumours rarely response to anti-PD-L1/PD-1 therapy. This classification is based on whether the tumours harbour an inflammatory microenvironment (11).

PD-1/1L was proved to reflect the activity of effector $\mathrm{T}$ cells and correlated with response rate to AntiPD-1/ PD-L1 therapy. Clinical trials such as CheckMate-012, KEYNOTE-001, and OAK further demonstrate that antiPD-1/L1 as a biomarker with strong predictive power is quite reliable now $(6,12,13)$. KEYNOTE-024 set the inclusion criteria to patients who had previously untreated advanced NSCLC with PD-L1 expression on at least 50\% of tumor cells, and the response rate reached $44.8 \%$ in the pembrolizumab treatment group (4).

Concerning all the existing data and economic factors, the expert panel recommend that PD-1/PD-1L to be a biomarker of single antiPD-1/PD-L1 treatment despite that the expression of PD-1/L1 may be diverse subjected to the time, site and treatment exposure. TIL, mutational load or neo-antigen burden, peripheral blood markers and immune gene signatures should be further explored to complete the prediction method for checkpoint blocks in multiple dimensions.

\section{Update}

Nowadays the detection of PD-L1 levels by using immunohistochemistry (IHC) staining is most commonly applied in clinical practice, but its prediction for response to antiPD-1/PD-L1 is imperfect as the dynamic and unstable expression. Then various biomarkers are developed to identified the heterogeneous tumor immune-environment, include TMB; the status of tumor infiltrating lymphocytes (TILS) and immunoscore; T-cell receptor clonality; DNA mismatch repair (MMR) deficiency, and several plasma biomarkers.

Some of these biomarkers are proposed to simplify the classification of immune status for clinical application. But the biological complexity of the tumor and immune system interaction make it impossible to use single biomarker to predict the response of ICIs. Another limitation of such biomarkers is that they are designed to represent key immune checkpoint proteins level instead of patients' individual immune status.

To consult such limitations and complete the evaluation system, several multivariate prediction models are set up. A personalized cancer medicine company named Mitra biotech even designed an ex vivo tumor model, which use tumor tissues to simulate the interaction between checkpoint inhibitors and tumor cells. By providing a real-time analysis of the tumor-immune status under the ICIs treatment, this evaluation system can not only identify the primary resistance groups but also can guide the following system therapy when patients acquired the resistance. Laura Mezquita et al. developed a Lung Immune Prognostic Index based on Derived neutrophils/ (leukocytes minus neutrophils) ratio (dNLR) and peripheral lactate dehydrogenase $(\mathrm{LDH})$ concentration, which can predict the survival benefit pretreatment.

Individual response to checkpoint blocks prediction based on a more comprehensive genotyping, prognostic stratification through more informative tumor immune environment are ushering in an exciting new era of precision medicine for patients with lung cancer. Recent developments in research and technologies have facilitated better understanding of this interaction and will provide means for development of better biomarkers. But each of the potential biomarkers should be validated carefully before clinical application.

\section{Consensus 4}

\section{More attentions should be paid to side effects caused by checkpoint inbibitors especially among elder patients}

\section{Level 2A}

Nowadays, checkpoint blocks are described as fantastic agents which can bring excellent response rate among multi-tumors with fewer side effects. As most clinical trials are still ongoing, application of checkpoint inhibitors should be cautious before a more reasonable comprehensive understand of this therapy was set up. PD-1/PD-L1 axis plays an important role to maintain immune homeostasis. The antiPD-1/PD-L1 therapy may break the immune self-tolerance and causes damage to normal issues such as respiration system, digestive system and nervous system.

Clinical Data have already demonstrated that checkpoints blocks can induce various immune-related adverse events (irAEs). Nearly $50 \%$ of patients will come up with rash and mucosal irritation during the treatment with Ipilimumab (14). Diarrhea is another common adverse effect (30\%) most often occurred among patients undergoing CTLA-4 blockade, and less than $10 \%$ will suffer grade $3 / 4$ diarrhea (15). Several trials also reported that PD-1 blocks can cause hepatotoxicity in less than $10 \%$ of patients causing the elevations in aspartate aminotransferase, aminotransferase (16-18). Endocrinopathy also occurred within $10 \%$ 
of patients undergoing CTLA-4 blockade treatment, among which hypophysitis and hypothyroidism are most common (19).

Besides the general adverse effects above, recently studies show checkpoint blockades even can accelerate the development of tumor. Based on the medical records from patients prospectively treated by anti PD-1/PD-L1 agents, Professor Champiat finds a novel aggressive pattern of hyper-progression exists in a subset of patients treated by checkpoint blocks. A total of $9 \%$ of patients included in this trail suffered hyper-progression disease (HPD, defined as $a \geq 2$-fold increase of disease progression). Further analysis demonstrates a significant correlation between the risk of HPD and age which may due to a different immunological background in older patients, HPD status was observed in $19 \%(7 / 36)$ patients older than 65 after antiPD-1/PDL1 treatment. Despite the limitation of sample size, this research suggests the application of anti-PD-1/L1 agents among elder patients especially older than 65 should be more cautions (20).

As the mechanisms of such adverse effects are far from fully understood, some rare side effects should also be taken into consideration when conducting the checkpoint inhibitors. In one case report published in American Fournal of Case Reports, Professor Jiro Abe described a 58-year-old male with a recurrence of lung adenocarcinoma suffered the akathisia which presented with unbearable restlessness and distress during the treatment of nivolumab. Akathisia is unlikely to response to traditional therapy and this patient finally came to need deep sedation (21). Another serious adverse effect caused by checkpoint blocks are reported by professor Douglas B. Johnson, the brief report published in The New England fournal of Medicine presents two patients with melanoma in whom fatal myocarditis developed after treatment with ipilimumab and nivolumab. Concerning the characters of immune-mediated myocarditis such as early onset, nonspecific symptomatology, and fulminant progression, clinical application of combine immunotherapy (ipilimumab and nivolumab) should be more cautious, and thus monitoring strategy should be worked out to reduce the risk (22).

Based on the clinical data, the expert panel recommend that more attentions should be paid to side effects caused by Checkpoint inhibitors especially among elder patients $(\geq 65$ years old).

\section{Update}

ICIs have broaden the therapy spectrum for lung cancer and brought remarkable clinical benefits, but the following irAEs are continuously reported within ICIs' increasing application in clinical practice. As the disorder of immune function caused by ICIs can derive systemic damage, irAEs are usually classified by organ system. IrAEs commonly occurred in gastrointestinal, dermatological, and endocrine systems. Among all irAEs, diarrhea, colitis, and/or autoimmune hepatotoxicity are the most common side-effects. It's not quite common but more serious when it comes to pulmonary, neurologic, hematologic, and cardiac systems.

IrAEs may interrupt the continuous therapy and even cause fatal adverse events, early detection and intervention are imperative for patients under the treatment of ICIs. Our consensus has highlighted the function of age to be an independent predictor for adverse events. Other predictions included immune cells levels, neoantigen, individual characters such as pharmacologic responses, microbiome and co-morbidities also can help to identify the potential groups who would fail to benefit from ICIs owing to accompanied adverse effects. Recently, one study reported the relationship between circulating B cell abundance and IrAEs risk, and the monitor of peripheral B cell could guide earlier clinical intervention and relief further serious adverse events.

With proper management, most irAEs would restore in a short time. However, no guidelines have been published regarding the management of irAEs till now. The common treatment for such adverse events are nonspecific symptomatic treatment, including corticosteroids or immunosuppressant. As peripheral immune cells levels have an association with the risk of irAEs, Tocilizumab targeted to IL-6 receptor are tested to be a safe remedy with marked curative effect.

IrAEs are not always bad news for patients treated with ICIs. Studies have demonstrated that a better survival benefits are associated with irAEs both in melanoma and lung cancer patients. To guarantee the effect of ICIs, a convinced and established guideline for ICIs management which is supported by strict clinical trials are in urgent need.

\section{Consensus 5}

\section{Exploring the strategy to overcome resistance to checkpoint inbibitors is in urgency}

\section{Level 2A}

Despite excellent clinical benefits brought by checkpoint blocks among multi-tumors, majority patients failed the treatment because of primary resistance. Along with the application of immunotherapy in more patients, remarkable 
treatment efficacy of checkpoint inhibitors also will be restricted as the development of acquired resistance. To overcome the limitation on checkpoint blocks requires further understanding of the molecular mechanisms of resistance to immunotherapy. Exploring actionable strategies to prevent the resistance not only can broaden clinical applicability of checkpoint blocks, but also can hold the long-lasting responses.

As resistance to immunotherapies may manifest at different times during the treatment, professor Padmanee Sharma proposed a brief summarization, which divide the resistance mechanisms into three main categories (23). Mechanisms of primary resistance exist at the time of initial presentation, which would lead to patients' unresponsive to the initial treatment. As the evolving nature of tumors, primary resistance could evolve into adaptive immune resistance after the treatment of checkpoint inhibitors. Acquired resistance occurs when tumor cells obtain the ability to resist the activity of checkpoints inhibitors to which it was previously response. As the broaden application of checkpoint blocks, data from KEYNOTE-006 has demonstrated that approximately one fourth to one third of patients who initially have objective responses to antiCTLA-4 or anti-PD-1 relapsed at last (24).

The resistance to checkpoint inhibitors can be caused by factors from either tumor-cell-intrinsic or -extrinsic. Based the data of studies conducted till now, the tumorcell-intrinsic factors mainly contains the signal pathway of mitogen-activated protein kinase (MAPK), the expression of the WNT/b-catenin, loss of interferon-gamma (IFN- $\gamma$ ) signaling pathways, and loss of tumor antigen expression (25-27). On the other side, tumor-cell-extrinsic members that lead to resistance including regulatory $\mathrm{T}$ cell (Tregs), myeloid derived suppressor cells (MDSCs), M2 phenotype tumor associated macrophages, and other inhibitory immune checkpoints $(28,29)$. Further study is undergoing to invest potential resistance mechanisms which can provide the basis for later discovering potential solutions.

Based on the study of intrinsic mechanism i immunotherapy, several strategies have been worked out to reverse the resistance to checkpoint blocks. Amounts of efforts have been made to transform immunologically "cold" tumors into "hot" tumors, among of which indoleamine 2,3-dioxygenase and some other potential targets were discovered (30). The most common tactics under conduction are the development of potential combination schedule of exist therapies, and several clinical trials are under conducting. The combine of antiPD-1/ PD-L1 and antiCTLA-4 have achieved higher response rate among patients with melanoma compared with single agent therapy $(31,32)$.

Considering the majority patients failed the treatment because of primary resistance and approximately one third of patients who initially have objective responses to checkpoint blocks relapsed at last. The expert panel supposed that exploring strategies to overcome resistance to the checkpoint inhibitors is in urgency, and more clinical trials should be conducted to test the potential strategies.

\section{Update}

Nowadays, most of studies on resistance to ICIs are focus on identifying patients who would failed therapy at the beginning. Based on the recently studies, Lower TMB, nondetectable PD-L1 expression are the most often described reasons for de-novo resistance.

At the same time, mechanisms of acquired resistance ICIs are far from understood. Neoantigen loss and tumor autonomous PD-1L level alternation caused by IFN signal pathway has been proposed to interpret the formation of acquired resistance. Besides, Gettinger reported an investigation based on 14 ICI-resistant lung cancer samples suggest that HLA Class I antigen processing and presentation machinery (APM) disruption can induce the resistance to ICIs during the treatment in lung cancer.

Additional studies are still under conduction to identify individual immune elements impeding the ICIs, and many researchers put a lot efforts in exploring further solutions based on such potential mechanisms. Till now, the most common strategy studied underway is to combine immunotherapeutic agents with targeted agents, cytotoxic chemotherapy, and/or radiation.

Within the clinical application progress of ICIs, more patients failed such therapy during the treatment will be reported continually. A system management of resistance to ICIs are needed. Besides the development of clinical system management, fresh exploration deep into mechanism how tumor cell escape from immune cytotoxic effects also can help to broaden the spectrum of ICIs.

\section{Acknowledgements}

None.

\section{Footnote}

Conflicts of Interest: The authors have no conflicts of interest to declare. 


\section{References}

1. Rosenberg SA, Yang JC, Restifo NP. Cancer immunotherapy: moving beyond current vaccines. Nat Med 2004;10:909-15.

2. Butts C, Socinski MA, Mitchell PL, et al. Tecemotide (L-BLP25) versus placebo after chemoradiotherapy for stage III non-small-cell lung cancer (START): a randomised, double-blind, phase 3 trial. Lancet Oncol 2014;15:59-68.

3. Tyagi P, Mirakhur B. MAGRIT: the largest-ever phase III lung cancer trial aims to establish a novel tumor-specific approach to therapy. Clin Lung Cancer 2009;10:371-4.

4. Reck M, Rodriguez-Abreu D, Robinson AG, et al. Pembrolizumab versus Chemotherapy for PD-L1Positive Non-Small-Cell Lung Cancer. N Engl J Med 2016;375:1823-33.

5. Dasanu CA, Sethi N, Ahmed N. Immune alterations and emerging immunotherapeutic approaches in lung cancer. Expert Opin Biol Ther 2012;12:923-37.

6. Herbst RS, Baas P, Kim DW, et al. Pembrolizumab versus docetaxel for previously treated, PD-L1-positive, advanced non-small-cell lung cancer (KEYNOTE-010): a randomised controlled trial. Lancet 2016;387:1540-50.

7. Gralla RJ, Spigel D, Bennett B, et al. PD1.01 (also presented as P2.46): LCSS as a Marker of Treatment Benefit With Nivolumab vs Docetaxel in Pts With Advanced Non-Squamous NSCLC From Checkmate 057. J Thorac Oncol 2016;11:S171.

8. Gainor JF, Shaw AT, Sequist LV, et al. EGFR Mutations and ALK Rearrangements Are Associated with Low Response Rates to PD-1 Pathway Blockade in Non-Small Cell Lung Cancer: A Retrospective Analysis. Clin Cancer Res 2016;22:4585-93.

9. Jänne PA, Yang JC, Kim DW, et al. AZD9291 in EGFR inhibitor-resistant non-small-cell lung cancer. N Engl J Med 2015;372:1689-99.

10. Teng MW, Ngiow SF, Ribas A, et al. Classifying Cancers Based on T-cell Infiltration and PD-L1. Cancer Res 2015;75:2139-45.

11. Chen DS, Mellman I. Elements of cancer immunity and the cancer-immune set point. Nature 2017;541:321-30.

12. Gettinger S, Rizvi NA, Chow LQ, et al. Nivolumab Monotherapy for First-Line Treatment of Advanced NonSmall-Cell Lung Cancer. J Clin Oncol 2016;34:2980-7.

13. Rittmeyer A, Barlesi F, Waterkamp D, et al. Atezolizumab versus docetaxel in patients with previously treated non-small-cell lung cancer (OAK): a phase 3, open- label, multicentre randomised controlled trial. Lancet 2017;389:255-65.

14. Lacouture ME, Wolchok JD, Yosipovitch G, et al. Ipilimumab in patients with cancer and the management of dermatologic adverse events. J Am Acad Dermatol 2014;71:161-9.

15. Hodi FS, O'Day SJ, McDermott DF, et al. Improved survival with ipilimumab in patients with metastatic melanoma. N Engl J Med 2010;363:711-23.

16. Robert C, Long GV, Brady B, et al. Nivolumab in previously untreated melanoma without BRAF mutation. N Engl J Med 2015;372:320-30.

17. Topalian SL, Sznol M, McDermott DF, et al. Survival, durable tumor remission, and long-term safety in patients with advanced melanoma receiving nivolumab. J Clin Oncol 2014;32:1020-30.

18. Hamid O, Robert C, Daud A, et al. Safety and tumor responses with lambrolizumab (anti-PD-1) in melanoma. N Engl J Med 2013;369:134-44.

19. Corsello SM, Barnabei A, Marchetti P, et al. Endocrine side effects induced by immune checkpoint inhibitors. J Clin Endocrinol Metab 2013;98:1361-75.

20. Champiat S, Dercle L, Ammari S, et al. Hyperprogressive Disease Is a New Pattern of Progression in Cancer Patients Treated by Anti-PD-1/PD-L1. Clinical Cancer Research 2017;23:1920-8.

21. Abe J, Sato T, Tanaka R, et al. Nivolumab-Induced Severe Akathisia in an Advanced Lung Cancer Patient. Am J Case Rep 2016;17:880-2.

22. Johnson DB, Balko JM, Compton ML, et al. Fulminant Myocarditis with Combination Immune Checkpoint Blockade. N Engl J Med 2016;375:1749-55.

23. Sharma P, Hu-Lieskovan S, Wargo JA, et al. Primary, Adaptive, and Acquired Resistance to Cancer Immunotherapy. Cell 2017;168:707-23.

24. Robert C, Schachter J, Long GV, et al. Pembrolizumab versus Ipilimumab in Advanced Melanoma. N Engl J Med 2015;372:2521-32.

25. Liu $\mathrm{C}$, Peng $\mathrm{W}, \mathrm{Xu} \mathrm{C}$, et al. BRAF inhibition increases tumor infiltration by $\mathrm{T}$ cells and enhances the antitumor activity of adoptive immunotherapy in mice. Clin Cancer Res 2013;19:393-403.

26. Spranger S, Bao R, Gajewski TF. Melanoma-intrinsic betacatenin signalling prevents anti-tumour immunity. Nature 2015;523:231-5.

27. Gao J, Shi LZ, Zhao H, et al. Loss of IFN-gamma Pathway Genes in Tumor Cells as a Mechanism of Resistance to Anti-CTLA-4 Therapy. Cell 2016;167:397-404.e9. 
28. Rudensky AY. Regulatory T Cells and Foxp3. Immunol Rev 2011;241:260-8.

29. Bronte V, Wang M, Overwijk WW, et al. Apoptotic Death of CD8+ T Lymphocytes After Immunization: Induction of a Suppressive Population of Mac-1+/Gr-1+ Cells. J Immunol 1998;161:5313-20.

30. Gubin MM, Zhang X, Schuster H, et al. Checkpoint Blockade Cancer Immunotherapy Targets Tumour-

Cite this article as: $\mathrm{Wu} \mathrm{YL}$, Wang CL, Liao ML, Guan ZZ, Gao CY, Lu S, Zhao MF, Wang J, Liu XQ, Yang JJ, Liang J, Mao WM, Han BH, Zhang XC, Song Y, Feng JF, Ma SL, Wu G, Zhou CC, Chen KN, Cheng Y, He Y, Chen C, Wang Q, Lin JZ, Zhu B, Liu YP, Hu Y, Qiao GB, Zhou Q, Song QB, Wu N, Wu L, Huang C, Fu XL, Xiong JP, Hu J, Hu CP, Chang JH, Zhao Q, Zhao J, Zhou PH, Ma ZY, Chen Y, Zhang HL, Yang F, Wang JJ, Pan YY, Yang XN, Fan Y, Liu Z, Fan W, Yang N, Guan YF, Sun H, Zhong WZ. A consensus on immunotherapy from the 2017 Chinese Lung Cancer Summit expert panel. Transl Lung Cancer Res 2018;7(3):428-436. doi: 10.21037/ tlcr.2018.04.15
Specific Mutant Antigens. Nature 2014;515:577-81.

31. Larkin J, Chiarion-Sileni V, Gonzalez R, et al. Combined Nivolumab and Ipilimumab or Monotherapy in Untreated Melanoma. N Engl J Med 2015;373:23-34.

32. Postow MA, Chesney J, Pavlick AC, et al. Nivolumab and Ipilimumab versus Ipilimumab in Untreated Melanoma. N Engl J Med 2015;372:2006-17. 